\title{
FAR-OUTER GALAXY MOLECULAR CLOUDS
}

And a comparison of molecular clouds across the Galaxy

\section{J. BRAND}

Istituto di Radioastronomia, CNR

Via Gobetti 101, 40129 Bologna, ITALY

AND

J.G.A. WOUTERLOOT

I. Physikalisches Institut der Univ. zu Köln

Zülpicher Str. 77, 50937 Köln, GERMANY

\section{Introduction}

The outer Galaxy (defined as those parts of the Galaxy with galactocentric distances $R>R_{0}=8.5 \mathrm{kpc}$ ) in general and particularly the outermost regions ( $\mathrm{R} \gtrsim 16 \mathrm{kpc}$; the far-outer Galaxy or FOG) has not received the attention that has been dedicated to the inner Galaxy and the solar neighbourhood.

It is however important to identify and study molecular clouds and embedded star forming regions also at large $\mathrm{R}$, in order to extend our understanding of the processes that lead to the formation of clouds and stars under physical conditions that are quite different from those found in the solar neighbourhood and in the inner Galaxy.

In very general terms one could say that most things that one finds in the inner Galaxy are also present in the far-outer Galaxy, but there is much less of it: The $\mathrm{HI}$ and $\mathrm{H}_{2}$ gas surface and volume densities are much smaller, and their scale-heights are larger; the star density is much smaller, there is less star formation; the interstellar radiation field (ISRF) is much weaker; there are no (known) spiral arms and fewer SNR's, so there are fewer and/or weaker external triggers for star formation; He and metal abundances are lower; the FIR emissivity is smaller, and the pressure of the intercloud medium is much smaller.

In a series of papers (Refs. [1]-[7]) we have established an observational data base of molecular clouds in the FOG, and of the detectable manifestations of star formation that goes on within them. 


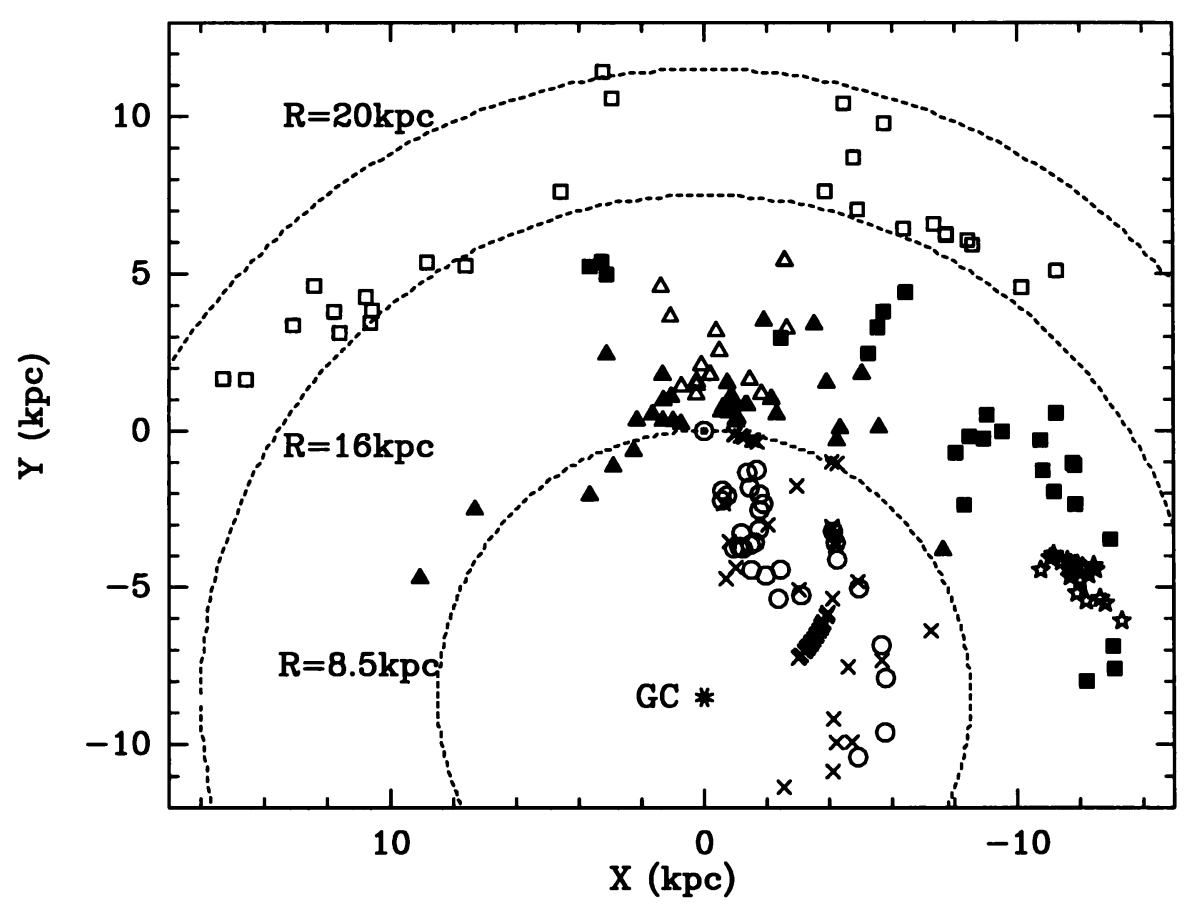

Figure 1. Distribution of the molecular clouds used in the analysis. The Sun is at $(\mathrm{X}, \mathrm{Y})=(0,0)$ and indicated by $\odot$; the galactic center is at $(\mathrm{X}, \mathrm{Y})=(0,-8.5)$. Dashed circle segments indicate $R=8.5,16$, and $20 \mathrm{kpc}$ respectively. The individual data sets are distinguished by different symbols: [5] (open squares), [8] (filled squares), [9] (open triangles), [10] (filled triangles), [11] (asterisks), [12] (circles), [13] (crosses).

\section{Data Base}

A general survey to locate sites of star formation in the outer Galaxy was made by WB89 [2], who colour-selected IRAS sources with a FIR spectral distribution typical of star-forming regions from the Point Source Catalogue, and used them as tracers for the molecular gas. For $\mathrm{R}>8.5 \mathrm{kpc}$ more than 1000 molecular clouds with embedded IRAS sources were found, of which almost 200 are at $\mathrm{R}>14 \mathrm{kpc}$. BW94 [5] mapped 27 clouds from the WB89 sample in ${ }^{12} \mathrm{CO}(1-0)$, and observed several other $\mathrm{CO}$ isotopes and -transitions at selected positions. The clouds in this sample are at $15.7 \mathrm{kpc}<\mathrm{R}_{\text {kin }}<20.2 \mathrm{kpc}$, with an average $\mathrm{R}$ of $17.3 \pm 1.2 \mathrm{kpc}(1 \sigma)$; the median value is $16.8 \mathrm{kpc}$. We adopt $R=17 \mathrm{kpc}$ as a representative distance.

We analyze these data, and then compare the results with those derived 


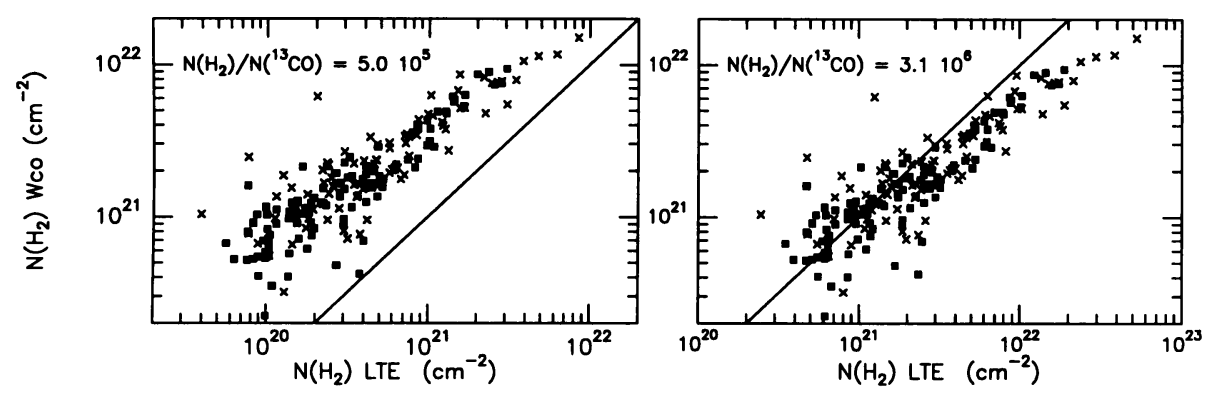

Figure 2. Comparison of column densities of $\mathrm{H}_{2}$ calculated via $\mathrm{N}_{\mathrm{Wco}}=\mathrm{X} \mathrm{W}_{\mathrm{CO}}$, with $\mathrm{N}_{\text {lte }}$. The filled squares are IRAM 30-m data of the WB283 cloud and the crosses SEST data of several other FOG clouds. Left-hand panel: used local abundance ratio $\mathrm{N}\left(\mathrm{H}_{2}\right) / \mathrm{N}\left({ }^{13} \mathrm{CO}\right)$; right-hand panel: used abundance ratio at $R=17 \mathrm{kpc}$, extrapolated from the gradients given by [16]. In both panels $\mathrm{X}=2.310^{20} \mathrm{~cm}^{-2}\left(\mathrm{Kkms}^{-1}\right)^{-1}$, i.e. the value appropriate for the inner Galaxy, and the drawn line is for equal column densities. When taking the abundance gradients into account $\mathrm{N}_{\mathrm{Wco}} \approx \mathrm{N}_{\text {lte }}$ for $\mathrm{X}$ in the far-outer Galaxy equal to the inner Galaxy value.

from similar observations of inner- and outer Galaxy clouds taken from the literature ([8]-[13]), and we try to use this to quantify differences (if any) in the various physical parameters (e.g. masses, kinetic temperature, and molecular abundances) between the inner- and far-outer Galaxy that could be a result of the changing environment. Figure 1 shows the galactic distribution of all molecular clouds used in the analysis. Including the literature data, we have an extended sample with parameters of 222 molecular clouds with $3<\mathrm{R}<20 \mathrm{kpc}$, of which 130 are at $\mathrm{R}>8.5 \mathrm{kpc}$. To allow a meaningful comparison we scaled kinematic distances with the same rotation curve, $\mathrm{X}\left(=\mathrm{N}\left(\mathrm{H}_{2}\right) / \mathrm{W}_{\mathrm{CO}}\right)$ to $2.310^{20} \mathrm{~cm}^{-2}\left(\mathrm{~K} \mathrm{kms}^{-1}\right)^{-1}$, and virial mass $\mathrm{M}_{\mathrm{vir}}$ to $126 \mathrm{r} \Delta \mathrm{v}^{2}$.

\section{Conversion Factor $\mathbf{X}=\mathbf{N}\left(\mathbf{H}_{2}\right) / \mathbf{W}_{\mathrm{CO}}$}

BW94 have mapped their clouds completely in ${ }^{12} \mathrm{CO}(1-0)$, and also the majority of the literature data are of ${ }^{12} \mathrm{CO}(1-0)$ only. For the derivation of cloud masses we therefore depend on so-called "W $\mathrm{CO}_{\mathrm{CO}}$-masses", obtained from the integrated intensity $\mathrm{W}_{\mathrm{CO}} \equiv \int \mathrm{Tdv}$, and the empirical fact that in the inner Galaxy there is a constant ratio $\mathrm{X}=\mathrm{N}\left(\mathrm{H}_{2}\right) / \mathrm{W}_{\mathrm{CO}} \approx(2.3 \pm 0.3)$ $10^{20} \mathrm{~cm}^{-2}\left(\mathrm{Kkms}^{-1}\right)^{-1}$ [14]. The resulting column density we shall denote as $\mathrm{N}_{\mathrm{Wco}}$ and the mass derived from this as $\mathrm{M}_{\mathrm{Wco}}$.

For about 200 lines of sight towards molecular clouds at $\mathrm{R}>16 \mathrm{kpc}$ we have taken ${ }^{13} \mathrm{CO}$ data, and performed an LTE analysis to derive $\mathrm{H}_{2}$ column densities $\mathrm{N}_{\text {lte }}$. 

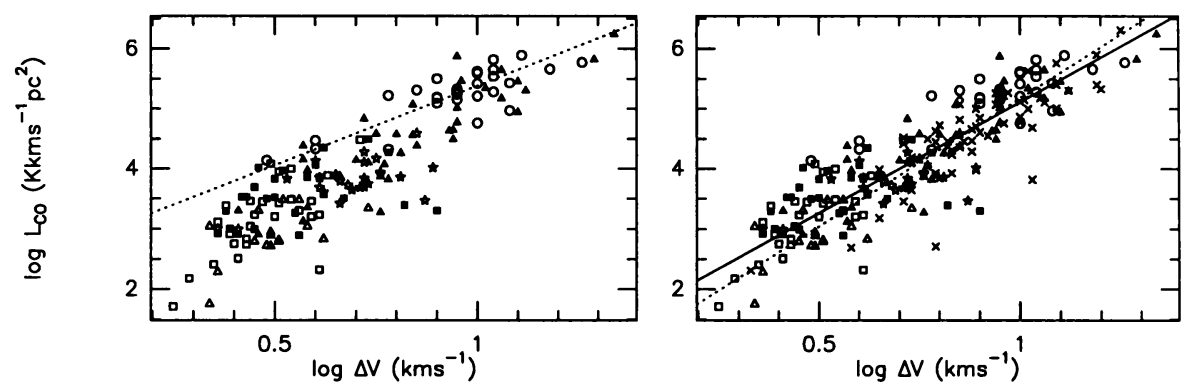

Figure 3. a. $\log \mathrm{L}_{\mathrm{CO}}$ versus $\log \Delta \mathrm{v}$ for all outer Galaxy clouds from the extended sample and the inner Galaxy clouds from [12]. The dashed line is a least-squares fit through the latter points. Symbols are as in Fig. 1. b. The same as a., but showing all inner Galaxy clouds $[12,13]$. The drawn line is a least-squares fit through the outer Galaxy data only; the dashed line fits the inner Galaxy data.

Figure 2 (left) shows a plot of $\mathrm{N}_{\mathrm{Wco}}$ versus $\mathrm{N}_{\text {lte }}$, which has been calculated using $\mathrm{N}\left(\mathrm{H}_{2}\right) / \mathrm{N}\left({ }^{13} \mathrm{CO}\right)=5.010^{5}$ (the value determined by [15] for local dark clouds). The drawn line indicates equal column densities. We see that $\mathrm{N}_{\text {lte }}<\mathrm{N}_{\mathrm{Wco}}$; the two can be made to agree by either reducing

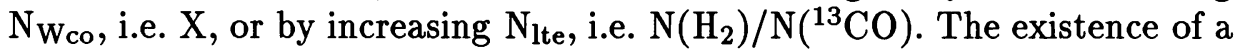
galactic abundance gradient is an observational fact, and the second option is to be preferred. Extrapolating the observed and modeled [16] abundance gradients to $\mathrm{R}=17 \mathrm{kpc}$ we estimate $\mathrm{N}\left(\mathrm{H}_{2}\right) / \mathrm{N}\left({ }^{13} \mathrm{CO}\right)=3.110^{6}$, i.e. ${ }^{13} \mathrm{CO}$ abundance 6 times lower than in the solar neighbourhood. Adjusting $\mathrm{N}_{\text {lte }}$ accordingly results in a situation where the data points are seen to scatter around the line of equal column densities (right), except for larger $\mathrm{N}_{\text {lte }}$, which is most likely due to saturation of ${ }^{12} \mathrm{CO}$. Averaging over all 200 lines of sight gives $\mathrm{X}=\mathrm{N}_{\text {lte }} / \mathrm{W}_{\mathrm{CO}}=(3.3 \pm 1.7[1 \sigma]) 10^{20} \mathrm{~cm}^{-2}\left(\mathrm{Kkms}^{-1}\right)^{-1}$ (median $2.910^{20}$ ), i.e. within $30 \%$ of the inner Galaxy value.

Using the extrapolation of measured abundance gradients to the FOG one finds that using $X=2.310^{20}=$ constant results in $\mathrm{H}_{2}$ column densities close to $N_{\text {lte. }}$.

\section{Are FOG Clouds Underluminous?}

In marked contrast to our conclusion in section 3, it has been argued (e.g. [8-11]) that in the outer Galaxy the value of $\mathrm{X}$ is 2.5 to 4 times larger than in the inner Galaxy. This is based on a comparison of a small sample of inner- and outer Galaxy clouds in a $\log \mathrm{L}_{\mathrm{CO}}$ versus $\log \Delta \mathrm{v}$ diagram, in which the outer Galaxy clouds lie systematically lower. Because $\mathrm{M}_{\mathrm{Wco}} \propto \mathrm{X} \mathrm{L}_{\mathrm{CO}}$, and assuming $\mathrm{M}_{\mathrm{Wco}_{\mathrm{co}}}=\mathrm{M}_{\mathrm{vir}}$, it was concluded that $\mathrm{X}$ must be higher in 

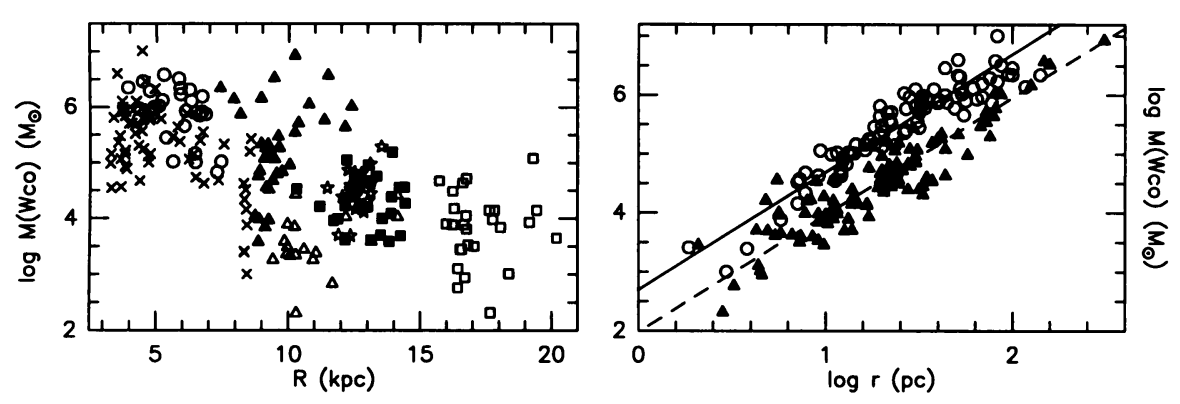

Figure 4. a. Plot of $\log \mathrm{M}_{\mathrm{W} c o}$ versus $\mathrm{R}$ for clouds in the extended sample. Symbols as in Fig. 1. b. Log $\mathrm{M}_{\mathrm{w}}$ co versus $\log \mathrm{r}(\mathrm{r}=$ cloud radius). Inner- (circles) and outer (triangles) Galaxy clouds define relations with the same slope (2.0) but are offset from one another such that at the same mass inner Galaxy clouds are half the size of outer Galaxy clouds.

the outer Galaxy.

In Figure 3a we show a plot of cloud luminosity versus line width for all 130 clouds in the extended sample (section 2) with $\mathrm{R}>8.5 \mathrm{kpc}$. Taking only the inner Galaxy data from [12] there does seem to be a considerable difference compared to the outer Galaxy. However, adding inner Galaxy clouds from [13], the difference disappears (Fig. 3b) and fits to inner- and outer Galaxy clouds are seen to overlap. A comparison between figs. 3a and $b$ suggests that the systematic offset in $3 a$ is is based on a lack of small (i.e. low mass and luminosity) clouds in that inner Galaxy sample and consequently on a biased inner Galaxy fit. Figure $3 \mathrm{~b}$ shows there is no need for a sudden change in $\mathrm{X}$ at $\mathrm{R}_{0}$, consistent with our earlier conclusion.

\section{Cloud Masses; Mass Spectrum}

Figure 4a shows the distribution of cloud mass with R. In the inner Galaxy clouds with mass $\lesssim 310^{4} \mathrm{M}_{\odot}$ are missing, probably as a result of confusion. Between $R \approx 3$ and $20 \mathrm{kpc}$ the mass of the most massive cloud at a particular $\mathrm{R}$ decreases by about a factor of 100 .

We have binned the data in mass, and assuming the mass distribution is the same at all $R$, we have combined the inner- and outer Galaxy samples at some intermediate mass and fitted a power-law to the resulting distribution. A fit to $\mathrm{M}_{\mathrm{Wco}} \geq 3.7510^{4} \mathrm{M}_{\odot}$ yields $\mathrm{dN} / \mathrm{dM}_{\mathrm{Wco}} \propto \mathrm{M}_{\mathrm{Wco}}{ }^{-1.90 \pm 0.11}$; a weighted fit to the outer Galaxy clouds alone gives a slope of $-1.74 \pm 0.05$. These mass spectra are consistent with those found for samples of local and inner Galaxy clouds, as well as with those found for clumps within star-forming clouds. With a total $\mathrm{H}_{2}$ mass in the outer Galaxy of $810^{8} \mathrm{M}_{\odot}$ [3] (including 
a $36 \%$ correction for $\mathrm{He}$ ), these mass spectra imply there are about 6000 star-forming molecular clouds at $\mathrm{R}>8.5 \mathrm{kpc}$.

In Figure $4 \mathrm{~b}$ we show the relation between $\mathrm{M}_{\mathrm{Wco}}$ and cloud radius for inner- and outer Galaxy clouds. Least-squares fits to both samples have the same slope but are offset from one another such that at the same $\mathrm{M}_{\mathrm{Wco}}$, inner Galaxy clouds are half the size of outer Galaxy clouds. Taking into account that $\mathrm{X}$ may differ slightly between the two samples (section 3), and that some inner Galaxy clouds may have artificially higher $\mathrm{M}_{\mathrm{Wco}}$ due to superposition of various clouds along the line of sight, part of the offset appears to be real. This may be caused by inner Galaxy clouds having a higher density, due for instance to increased ambient pressure.

\section{Cloud Temperatures}

For two FOG clouds for which we have observations in up to $5{ }^{12} \mathrm{CO}$ and ${ }^{13} \mathrm{CO}$ transitions we have derived a $\mathrm{T}_{\text {kin }}$ in the range $10-18 \mathrm{~K}$, using escape probability models. This is consistent with the $10-15 \mathrm{~K}$ found by [17] for inner Galaxy clouds that are not located in the molecular ring. In addition we find, from a statistical analysis of antenna temperatures $\mathrm{T}_{\mathrm{A}}^{*}$ of fore- and background clouds in [2], and of the envelopes of FOG clouds in [5], that the average $\mathrm{T}_{\mathrm{A}}^{*}\left[{ }^{12} \mathrm{CO}(1-0)\right]$ does not decrease with $\mathrm{R}$ between 9 and $16 \mathrm{kpc}$. This suggests that there is no significant gradient in $\mathrm{T}_{\text {kin }}$ in molecular clouds in this part of the Galaxy (see also [6]).

This work is supported in part by the Deutsche Forschungsgemeinschaft (DFG) through grant SFB-301.

\section{References}

[1] Wouterloot J.G.A., Brand J., Henkel C., 1988, A\&A 191, 323

[2] Wouterloot J.G.A., Brand J., 1989, A\&AS 80, 149 (WB89)

[3] Wouterloot J.G.A., Brand J., Burton W.B., Kwee K.K., 1990, A\&A 230, 21

[4] Wouterloot J.G.A., Brand J., Fiegle K., 1993, A\&AS 98, 589

[5] Brand J., Wouterloot J G.A., 1994, A\&AS 103, 503 (BW94)

[6] Brand J., Wouterloot J.G.A., 1995, A\&A submitted

[7] Fiegle K., Wouterloot J.G.A., Brand J., Winnewisser G., 1995, A\&A in press

[8] Mead K.N., Kutner M.L., 1988, ApJ 330, 399

[9] Carpenter J.M., Snell R.L., Schloerb F.P., 1990, ApJ 362, 147

[10] Sodroski J., 1991, ApJ 366, 95

[11] Digel S.W., 1991, Ph.D. Thesis, Harvard Univ.

[12] Dame T.M., Elmegreen B.G., Cohen R.S., Thaddeus P., 1986, ApJ 305, 892

[13] Solomon P.M., Rivolo A.R., Barrett J., Yahil A., 1987, ApJ 319, 730

[14] Strong A.W., Bloemen J.B.G.M., Dame T.M. et al., 1988, A\&A 207, 1

[15] Dickman R.L., 1978, ApJS 37, 407

[16] Wilson T.L., Matteucci F., 1992, A\&AR 4, 1

[17] Sanders D.B., Scoville N.Z., Tilanus R.P.J., Wang Z., Zhou S., 1993, in: Holt S.S. \& Verter F., Back to the Galaxy, p. 311 